\title{
Optimal order quantity and pricing decisions in single-period inventory systems
}

\author{
L. Hakan Polatogilu* \\ Bılkent Untverstty. Department of Industrial Engineering, Ankara, Turkel'
}

\begin{abstract}
In this paper, we consider sımulianeous pricing and procurement decisions associated with a ont-period pute inventory model under determunistic or probabilistic demand. We investigate the necessar, and sufficient conditions for an ( $\sigma, \Sigma)$ type policy to be optımal for the determination of the procurement quantity. We also show how the corresponding optimal price can be obtained.
\end{abstract}

\section{Introduction and literature review}

In this paper, we study the optimal procurement and pricing decisions in a single-product, one-period pure inventory system. We view this model as a building block of the multi-period model and attempt to characterize an optimal one-period inventow v control policy that would apply to the multi-period model under genera? assumptions.

Most inventory models are constructed under the assumption that the decisions of the vendor do not alter the ficmend pattern or the prire structure in the market during the planning horizon. This assumpticn is approximated in a perfectly competitive market where there is no pricing decision to make for the individual vendor. Under imperfect competition, however, the vendor exercises a degree of ranopdy $F^{n w e r}$ in the market and faces a downward sloping demand curve. He may set a price foi ints product but then he faces a demand level, go:erned by some probabiiity distribution, the expectei "inle of which is decreasing in price. At the beginning of the period, given the inventory position, his problem is to determine the procurement and pricing folicies which jointly maxin. ize the expected value of the one period profit.

A number of special cases of this modsl have been studied in the iiterature. These differ essentially in the way the demand process is represented. In the additive model, $X(p)=\bar{X}(p)+\varepsilon$ where $X(p)$ is the demand when the price is $p, \bar{X}(p)=\mathrm{E}[X(p)]$ and $\varepsilon$ is a random variable with a known distribution and $\mathrm{E}[\varepsilon]=0$. In the multiplication model, $X(p)=\bar{X}(p)+\varepsilon$ where $\mathrm{E}[t \mid=1$. In the riskless model, $X(p)=\bar{X}(p)$ so that the demand is represented by its expected value. This latter case serves both as a first order approximation an as : benchmark for the prouabulistic versinn of the norlel. Note that while the demand variance is price-independent in the additi $s$ model, in the multiplicative model it is a decreasing functicri of price, under the (natural) assumption that $\bar{X}(p)$ is decreasing in price.

Whitin [1] appears to have been the first to link price theory and inventory controi in d vict-period model. Later, Mills [2] and $v_{\text {,arlio }}$ nd Carr [3] studied the additive model. They derived the necessaly couditions for optimality and showed, under reasonable as junntions, that the optimal price under

*The author is gicteful to Prof I Şahın (Universıty of Wisconsin, Milwaukee) for his continual interest, helpful comments and tımely encourdgement during the preparation of this paper The author is currenis at the University of $W_{15 c o n s i n}$ Milwaukee, School of Business Administration, PO Box 742. Milwaukee, Wisconsin 53201, USA 
uncertainty is less than the optimal riskless price. This conclusion is reversed for the multiplicative model [3]. Zabel worked on the existence and uniqueness of the optimal solutions for the multiplicative [4] and additive [5] models. Young [6] also studied similar issues for a unified demand modal in which $X(p)$ is given by a mmbination of the additive and multiplicative forms. These studies indicate that existence could be shown under resirictive assumptions on $X(p)$. Uniqueness, on the other hand. requires further restrictıons, especially on the distribution of $\varepsilon$

It has been common practice in demand modeling to express random denuand as a combination of expected demand and a random term. The former has some form of price dependency while the latter is price independent. This synthesis has been used traditionally as a convenient tool to isolate the effects of uncertainty in the context of the theory of the firm. The disadvantage of this representation, however, is the structural restrictions it brings into the model. For instance, the additive model is restricted by a constant variance. Also it allows negative demands uniess the price value: are restricted. The multiplicalive model implies the curious restriction that the demand equals the produri of its expected value and : randoin term. As a result of this, variance of demand is the square of its expected value times the variance of the random term. Therefore, variance decreases at a rate faster than expected value and it approaches to zero at high prices.

We believe that there is a need to study the model under general demand uncertainty. It is essential to reveal the fundamental properties of the model, independent of the demand pattern. Especially, uniqueness conditions for optimality must be studied in a more general setting. In what follows, we introduce the basic model in Seciiun 2 and develop and analyze it in Section 3 . We then link the model to earler studies in Section 4 by considering additive and multiplicative demand as special cases.

\section{Basic modiel and assumptions}

The vendor is to make the best procurement and pr cing decisions to maximize his profit prior to the beginning of the periou. Inventory level befor ufdering is $l$. The amount procured, if any, is $q-i$. A random demand $X$ occurs during the period and at the end of the period the inventory level is reauced to $y-X$.

In this study, we consider the case where $i \geqslant 0$. For $i<0$, the one-period problem is initiated with an unknown history. That is, the following questions can not be accounted for unless we make assumptions. (1) What fraction of the backlog do we have to satisty? (2) At what price should we sell that fraction? (3) Do we deduct the backlog from the actual demand or not?

We assume that inventory costs are proportional to the period ending inventory level. We denote the unit holding, shortag? and procurement costs by $h, s$ and $c$, respectively. Wo also denote the fixed ondering cost by $\mathscr{K}$

In addition, we assurne that the price is bounded from below and above by $P_{1}$ and $P_{u}$, respectively, which are the price floor and price celling in d regulatory environment. We also assume that $P_{u}>c$ so that it is possible to make a profit by retailing.

In this study, we wrik with a finite demand process; that is:

$0 \leqslant X_{1}(p) \leqslant X \leqslant \lambda_{2}(p)<\infty$

where $X_{1}(p)$ and $X_{2}(p)$ are the lower and upper bounds on $X$, respectively, which are differentiable functious of $p$. We are also given the demand distribution $F(x: p)$ which is defined over $x \in(-\infty, \infty)$ and $p \in\left[P_{i}, P_{u}\right\}$. We shall restrict curselves orily to the continuous demand case, bearing in mind that similar analysis exists otherwise.

We assume that $\bar{X}(p)$ is a monotone decueasing function of $p$ on $(0, \infty)$ (if $p$ is confined to $\left[P_{i}, P_{u}\right]$, then we extend $\bar{X}(p)$ on $\left(0, P_{l}\right)$ and $\left(P_{u}, \infty\right)$ by appropriate functions te satisfy the requirements without loss of generality). Morenver. we require that $X(p)$ is $o(1 / m)$ as $p \rightarrow 0^{+}$and $p \rightarrow \infty$. This implies that the function $p \cdot \bar{X}(p)$ stanis at zero, first increases and eventually dies away. This function, which is denoted by $R(p)$, is called the riskless total revenue by Mills [7]. $R(p)$ is a positive valued, finite and 
differentiable function, which plays an important role in model development. It is shown in the Appendix that $R(p)$ is pseudoconcave on $(0, \infty)$ when $\bar{X}(p)$ is either a concave or convex decreasing function; it is also indicated that $R(p)$ is not pseudoconcave for all monotone decreasing $X(p)$ functions. We assume that $R(p)$ is unimodal; hence, there exists a unique finite price which maximizes $R(p)$.

\section{Mathematical model}

In this section we develop and inalyze the mathematical model under probabilistic demand for the determination of the optimal pric: and the beginning inventory level.

\subsection{Optimization problem}

Considering the representation introduced in Section 2, the profit function can be expressed as:

$\Pi(p, q)=M(p, q)-\mathscr{K} \cdot \delta(q-i)$

where $\delta(\cdot)$ is the Heavyside function and

$M(p, q)= \begin{cases}p \cdot q-c \cdot(q-i)-s \cdot(X-q) & q \leqslant X \leqslant X_{2}(p) \\ p \cdot X-c \cdot(q-i)-h \cdot(q-X) & X_{1}(p) \leqslant X \leqslant q\end{cases}$

where $X$ is the randorn demand. We can write the expected profit as:

$\| \bar{l}(p, q)=\mathbb{E}[\Pi(p, q)]=\bar{M}(\tilde{p}, q)-\mathscr{K} \cdot \delta(q-i)$

where

$M(p, g)=E[M(p, q)]=? \cdot Y(p)-c \cdot(q-1)-L(p, u)$

The first term in (4) is the riskless total revenue function. The second term is the procurement cost. The last term is the exprected loss f "icullon which is given by

$L(p, q)=(p+s) \cdot[\dot{X}(p)-q]+(p+s+h) \cdot \theta(p, q)$

where $\theta(p, q)$ is the oxpected leftovers, i.e.

$\theta(p, q)=\int_{\lambda(1, p)}^{q}(q-x) \cdot f(x ; p) \mathrm{d} x=\int_{x(p)}^{q} F(x ; p) \mathrm{d} x$

We assume that $\theta(p, q)$ is differentiable in $p$ for $q \geqslant 0$ Moreover, we nbrerve that $\theta(p, q)$ satısfies

$\Theta(p, q) \geqslant \max \{0, q-\bar{X}(p)\}$

and it is a convex, nondecreasing and differentiable function of $q$ for a given $p$.

From (4) and ( $s)$ it follows that

$\bar{M}(p, q)=p \cdot[q-\theta(p, q)]-c \cdot(q-l)-h \cdot \theta(p, q)-s \cdot[\bar{X}(p)-(q-\theta(p, q))]$

Therefore, $\bar{M}(p, q)$ is the expected net revenue, less the procurement cost, less the expected loolding cost, and less the expected shoutuge cost. At the cxnense of loosing intuition about its terms, we shall refer to $\bar{M}(p, q)$ in the scquel in :'se followime form.

$M(p, q)=(p+s-\imath, q-;, \bar{X}(p)-(p+s+h) \cdot \theta(p, q)+c \cdot i$

It is clear that, $\bar{M}(p, q)$ is continuous in $p$ on $\left[P_{i}, P_{u}\right]$ and in $q$ on $[0, \infty)$

Now, the optimization problem becomes

$\bar{\Pi}\left(p^{*}, q^{*}\right)=\operatorname{nax}_{p_{4}}\left\{\bar{\Pi}(p, q): q \in[l, \infty), p \in\left\{P_{,}, P_{u}\right]\right\}$ 
where $p^{*}$ and $q^{*}$ are the optimal values of the decision variables $p$ and $q$. For this problem we define the suboptimal function

$\bar{M}^{*}(q)=\max \left\{\bar{M}(p, q): p \in\left[P_{1}, P_{u}^{*}\right]\right\}=\bar{M}\left(p_{q}, q\right)$

where $p_{q}$ is the maximizer. Therefore, $\bar{M}^{m}(q)$ traces the best price trajectory over the $q$ range. Moreover, since $\bar{M}(p, q)$ is continuous in $p$ and $q, \bar{M}^{*}(q)$ becumes a continuous function of $q$.

In analy<ing (10) and (11), we need to consider first and second degree partial derivatives of $\bar{M}(p, q)$ with respect to $p$ and $q$, which are given by

$$
\begin{aligned}
& \frac{\partial \bar{M}(p, q)}{\partial p}=q-s \cdot \frac{\mathrm{d} \bar{X}(p)}{\mathrm{d} p}-\theta(p, q)-(p+s+h) \cdot \frac{\partial \Theta(p, q)}{\partial p} \\
& \frac{\partial^{2} \bar{M}(p, q)}{\partial p^{2}}=-s \cdot \frac{\mathrm{d}^{2} \bar{X}(p)}{\mathrm{d} p^{2}}-2 \cdot \frac{\partial \theta(p, q)}{\partial p}-(p+s+h) \cdot \frac{\partial^{2} \Theta(p, q)}{\partial p^{2}} \\
& \frac{\partial^{2} \bar{M}(p, q)}{\partial p \partial q}=1 \cdots \Gamma(q ; p)-(p+s+h) \cdot \frac{\partial F(q, p)}{\partial p} \\
& \frac{\partial \bar{M}(p, q)}{\partial q}=(p+s-c)-(p+s+h) \cdot \bar{F}(q ; p) \\
& \frac{\partial^{2} \bar{M}(p, q)}{\partial q^{2}}=-(p+s+h) \cdot f(q ; p) \leqslant 0
\end{aligned}
$$

From $(15)$ we conciucie that $\bar{M}(p, q)$ is $q$-concave on $(0, \infty)$. which refers to the newsboy problem setting. On the other hand, $(12)$ inultes tisat $p_{q}$ is independent of the procurement cost. In other words, the vendor is to maximize his expected profit given that he starts the period with $g$ units. The price dependence of $\bar{M}(n, q)$, however, is nor clear froni (12) or (13).

If $p_{q}$ is independent of $q$ (a boludary point solution or a constant), then it follows from (15) that $\bar{M}^{*}(q)$ is concave at that $q$. However, if $p_{q} \in\left(P_{1},{ }_{u}^{\prime}\right)$, then it must satisfy the first order condition

$\dot{\partial} \bar{M}(p, q) /\left.\partial p\right|_{p q}=0$

and the second order condition

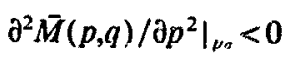

for a given $q$. Sinc $\bar{M}(p, q)$ has continuous partial derivatives " " can perform implicit differentiation on the first order condition to obtain

$\frac{\mathrm{d} p_{q}}{\mathrm{~d} q}=\frac{1-F\left(q ; p_{q}\right)-\left(p_{q}+s+h\right) \cdot \partial F(q, p) /\left.\mathrm{d} p\right|_{p_{q}}}{-\delta^{2} \bar{M}(p, q) /\left.\hat{v} p^{2}\right|_{p_{4}}}$

in which the denommator is always postive. Lependrs on the value of $p_{q}$ and the price dependency of $F(\cdot ; p)$ function, however, 'ile numerator can be po itive or negative Thus, the sign of $\mathrm{d} p_{o} / d q$ is hiut clear

Siner $\mathrm{d} \ddot{p}_{q} / \mathrm{d} q \mathrm{q}$ exists, wo can wite the inst derivative of $\bar{M}^{*}(q)$ as

$\frac{\mathrm{d} \bar{M}^{*}(q)}{\mathrm{d} g}=\frac{\partial \bar{M}\left(p_{r} \dot{1}\right)}{\partial q}+\left.\frac{\partial \bar{M}(p, q)}{\partial p}\right|_{p_{q}} \cdot \frac{\mathrm{d} p_{q}}{\mathrm{~d} q}$

If $p_{q} \in\left(P_{l}, P, j_{i}\right.$ then $\partial \bar{M}(p, y) /\left.\partial p\right|_{p_{\mathrm{g}}}=0$ otherwise $\mathrm{d} p_{\mathrm{q}} / \mathrm{d} y=0$. Theretore, 1 all combinations of rightrand and ieft hand derivatives the second term in (17) vanishes Conrsquently, we get 
$\frac{\mathrm{d} \bar{M}^{*}(q)}{\mathrm{d} q}=\left(p_{q}+s-c\right)-\left(p_{q}+s+h\right) \cdot F\left(q ; p_{q}\right)$

Furthermore, for $p_{q} \in\left(P_{l}, P_{u}\right)$, differentiating (18) with respect to $q$ we $r$ btain

$\frac{\mathrm{d}^{2} \bar{M}^{*}(q)}{\mathrm{d} q^{2}}=\frac{\mathrm{d} p_{q}}{\mathrm{~d} q} \cdot\left[1-F\left(q ; p_{q}\right)\right]-\left(p_{4}+s+h\right) \cdot \frac{\mathrm{d} F\left(q ; p_{q}\right)}{\mathrm{d} q}$

Noting that

$\frac{\mathrm{d} F\left(a ; p_{4}\right)}{\mathrm{d} q}=f\left(q ; p_{q}\right)+\left.\frac{\partial F\left(a ; n_{4}\right) !}{\partial p}\right|_{p_{q}} \cdot \frac{\mathrm{A} p_{q}}{\mathrm{~d} q}$

we tewrite (19) a's

$\frac{\mathrm{d}^{2} \bar{M}^{*}(q)}{\mathrm{d} q^{2}}=-\left.\frac{\partial^{2} \bar{M}(p, q)}{\partial p^{2}}\right|_{p_{q}} \cdot\left(\frac{\mathrm{d} p_{q}}{\mathrm{~d} q}\right)^{2}-\left(p_{q}+\varsigma+h\right) \cdot f\left(q ; p_{q}\right)$

The first term in (21) is al' magnitudes are not clear. Thus, convexity of $\bar{M}^{*}(q)$ is not evident from (21).

\subsection{Laisitice problem}

Intuitively, $\bar{M}^{*}(y)$ must have a peak on $[0, \infty)$. However, the existence of this pont or, if it exists, its location is not inmediately clear. In the following analysis, we shall identify two separate regions of $q$ in which $\vec{M}^{*}(q)$ is monotone, then we shall prove the existence of its peak.

Lemma 1. $\vee q \in\left[0, X_{1}\left(P_{u}\right)\right], \bar{M}^{*}(q)$ is a linear increasing funct on of $q$ and $p_{q}=P, \quad$.

Prowf. $\forall g \in\left[0, X_{1}\left(P_{u}\right)\right]$ we ilave $F\left(q ; p_{q}\right)=0$. Therefore, from $(6), \theta\left(p_{q}, q\right)=0$ and from $(9)$ we obtain:

$$
\begin{aligned}
\bar{M}^{*}(q) & =\max \left\{(p+s-c) \cdot q-s \cdot \bar{X}(p)+c \cdot i: p \in\left[P_{l}, P_{u}\right]\right\} \\
& =\left(P_{u}+s-c\right) \cdot q-s \cdot \bar{X}\left(P_{u}\right)+c \cdot \imath
\end{aligned}
$$

which 15 a linear increasing function of $q$ and $p_{q}=P_{u}$.

Lemma 1 ndiicatss that, if we are sure that demand will ex seed our stock, 1.e. if $q \leqslant X_{1}\left(P_{u}\right)$, then we should change the clistomeis at the hightest rate because we nct only reduce shortages in this way but we also obtain the maximum unit profit.

If $X_{1}\left(P_{u}\right)=0$, then the, regir.a indicated in Lemma 1 disappears and we lose the information about the slope of $M^{*}(q)$ at $q=0$. To account for this possibility, considering (18) and the fact that $0 \leqslant F\left(g: p_{q}\right) \leqslant 1$ we ontati:

$$
-(h+c) \leqslant \frac{\mathrm{d} \bar{M}^{*}(a)}{\mathrm{d}_{4}} \leqslant\left(p_{a}+s-c\right)
$$

which gives the lower and upper limits of the rate of change of expected profit with respect to the beginniug inventory level. It is now clear frcen (22) and (23) that at $q=0, \bar{M}^{*}(q)$ ircreases at the maximum raté of $P_{u}+s-c$.

Lemmat 2. $\forall q \in\left[\ddot{x}_{2}\left(P_{1}\right), \infty\right), \bar{M}^{*}(q)$ is a linear decrearing function of $q$ and $p_{q}$ is a constant. 
Proof, For $q>X,\left(P_{1}\right)$ we have $F\left(q ; p_{n}\right)=1$. The refore, from $(6), \Theta\left(p_{q}, q\right)=q-\bar{X}\left(r_{q}\right)$ and from $(\dot{y})$ we obtain

$$
\begin{aligned}
\bar{M}^{*}(g) & =\max \left\{(p+h) \cdot \bar{X}(p) \cdot p \in\left[P_{1}, P_{u} j\right\}-(c+h) \cdot q+c \cdot l\right. \\
& =\left(\vec{P}_{h}+h\right) \cdot \vec{X}\left(\vec{P}_{h}\right)-(c+h) \cdot \mathrm{q}-c \cdot i
\end{aligned}
$$

where $\bar{P}_{h}=\min \left\{\max \left\{P_{h}, P_{h}\right\}, P_{u}\right\}$ and $P_{h}$ is the maximizer of the pseudioconcave function $(p+h) \cdot \bar{X}(p)$.

We now establish the existence of $\tilde{q}$, wherc $\tilde{q}=\max \left\{\bar{T}^{*}(q) \cdot g \in[0, \infty)\right\}$.

Theorem 1. $\exists \tilde{q} \subset\left(Y_{1}\left(P_{w}\right), Y_{2}\left(P_{l}\right)\right)$ surh that $\bar{M}^{*}(q) \leqslant \bar{M}^{*}(\tilde{q}) \vee Q \in[0, \infty)$

Proot. By Lemma 1 , $i T^{t+}(q)$ is a lined ircreasing function of $q$ on $\left\{\eta, X_{1}\left(\mu_{u}\right)\right\}$ with a siope of $\left(P_{u}+s-c\right)=0$. By Lemına $2, \bar{M}^{*}(q)$ is a linear decreasing function of $q$ on $\left[X_{2}\left(P_{1}\right), \infty\right)$ with a slope of $-(c+h)<0$. From $(23),\left(P_{u}+s-c\right)$ and $-(c+h)$ are the largest and the smallest possible slopes of $\vec{M}^{*}(q)$, respectively. The proof follows.

Theretore, $q$ must satisfy the fit si urder optimally condition on $\bar{M}^{*}(q)$ which can be obtained from (18) as:

$F\left(q, p_{q}\right)=\frac{p_{q}+s-c}{p_{q}+s+h}$

RHS, the right hand side of (25), is a concave It foilows from (18) that, for those $p_{q}$ values $t$ price levei less than $c-s$. Alternatively, for $p_{q} \geqslant c-s, \mathbf{R}_{k}$ hove a solut:on fo: a guren such PHS

\section{Unimodalıy}

Unzmodalty of $\bar{M}^{*}(a)$ enables us to identify an $(\sigma, \Sigma)$ type policy which may be employed in determining the op:'mal $q$. Morenver, in the multiperiod extension of the theory, this becomes an essential ingredicnt of the dynamic decision problem.

it the vendor administers his profit maximizing price as he starts with a stock size of $q$, then $F\left(q ; p_{q}\right)$ represents the probability that the wil he no shortage. Note ttadt, $F\left(q ; p_{q}\right)$ is a function of $q$ only, where $F\left(q ; p_{q}\right)=0$ for $l \leqslant q \leqslant X_{i}\left(P_{u}\right)$ and $F\left(q ; p_{q}\right)=1$ for $X_{2}\left(p_{l}\right) \leqslant q$. Therefore, $F\left(q ; p_{q}\right.$, has to rise from 0 to 1 between minmum and maximum possuble demazid viues. Meanwhile, it is clear from Lemma and 2 that $p_{q}$ should decrease from $P_{u}$ to $\bar{P}_{n}$. If these char ${ }_{j}$ es occur monotonically, then there will be a unique fir,t order $q$, which satisfies $(23)$. That is, if $\mathrm{d}_{2}\left(q ; p_{q}\right) / \mathrm{d} q \geqslant 0$ and $\mathrm{dp}_{q} / \mathrm{d} q \leqslant 0$, then from (19) it tollow s that $\bar{M}^{*}(q)$ is concave. However, we can state a weaker condition by noting that $1 \mathrm{t}$ is sufficient to have $\mathrm{d} p_{q} / \mathrm{d} q \leq 0$ at $q=\hat{q}$. provided that $\mathrm{d} F\left(q ; p_{t}\right) / \mathrm{d} q \geqslant 0 \forall q$. That is,

$\frac{\mathrm{d} F(q, p}{\mathrm{d} q} \geqslant 0 \mathrm{a},\left.\mathrm{d} \frac{\mathrm{d} p_{q}}{\mathrm{~d} q}\right|_{q} \leqslant \mathrm{U} \Rightarrow \bar{M}^{*}(q)$ is uniriodal

Voreuvir from $(i 6)$ and $(25)$ we ootain

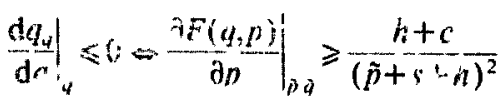


where $\tilde{p}=p_{q}$ and we can employ (27) in (26). On the other hand, we realize that for unimodality of $\bar{M}^{*}(q)$ it is necessary and sufficient to have

$\left.\frac{\mathrm{d}^{2} \bar{M}^{*}(q)}{\mathrm{d} q^{2}}\right|_{q} \leqslant 0$

\section{Optimal solution}

If $\bar{M}^{*}(q)$ is unimodal, then from $(10)$ it follows that $q^{*}$ can be determined by an $(\sigma, \Sigma)$ type policy operating on $\bar{M}^{*}(q)$, where $\Sigma=\tilde{q}$ and $\Sigma=\min \left\{q . \bar{M}^{*}(q)=\bar{M}^{*}(\Sigma)-\mathscr{H}\right\}$. Consequently, the decision ruie is $q^{*}=\Sigma$ if $<\sigma$, otherwise $q^{*}=l$, and

$p^{*}-\operatorname{argmax}\left\{\bar{M}\left(p, q^{*}\right): p \in\left[n_{1}, r_{u}\right]\right\}$

\section{Special cases}

In this section, first we cunsider the deterministic demand model (the riskless model introduced by Mills (21) and establish its relation to the probabilistic morlel. Then, we analyze the additive and the multuplicative models. We provide the rclationships that exist betwecn the opimal prices of these models. Finally, under linear expected demand $\left(\bar{X}(p)=a-b \cdot p\right.$, whice $a, b>0$ and $\left.c<P_{u}<a / b\right)$, we prove the unimodaiity of $\vec{M}^{*}(q)$ for uniformly distributed additive alini for exponentially distributed maltiplicative 2 .

\section{I neterministic model}

In this part, we use th s. "ubscript " $r$ " to denote the functions, and varables of the riskless model. If

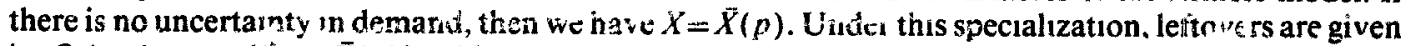
by $\theta_{r}(p, q) \sim \max \{\hat{U}, q-\overline{\bar{\lambda}}(p)\}$, whic $h$ is a contınuous function. It is, however, non differentiable at the trajectory given by $q=\bar{X}(p)$.

In the following discussion, first we prove that $M_{\mathrm{r}}^{*}(q)$ is unımodal, then we determine the optimal values of the decision variables, and finaily we compare the deterministic and nubabilstic profit functions.

Theorem 2. $M_{\mathrm{r}}^{*}(q)$ is quasiconcave in $q$ on $[0,(\infty)$.

Preof For $q \leqslant \tilde{X}\left(P_{u}\right)$ we have $\Theta_{1}(p, q)=0$. Thus, from Lemma 1 it follows that $M_{\mathrm{r}}^{*}(q)$ is a linear increasing function of $q$ and $p_{4}=P_{\ldots}$

For $\bar{X}\left(P_{u}\right) \leqslant q$ we define $\bar{p}$ such that $\bar{X}(\bar{p})=\min \left\{q, X\left(P_{i}\right)\right\}$. 1 herefore,

$\vartheta_{\mathrm{r}}(p, q)=0$ if $P_{l} \leqslant F \leqslant \bar{p}$

or

$\Theta_{\mathrm{r}}(p, q)=q-\bar{X}(p)$ if $\bar{p} \leqslant p \leqslant P_{u}$

Under this settıng, by Lemma 1 wc have

$\max \left\{M_{\mathrm{r}}(p, q): P_{1} \leqslant p \leqslant \bar{p}\right\}=M_{1}(p, q)$

Thus,

$M_{\mathrm{r}}^{*}(q)=\max \left\{M_{\mathrm{r}}(p, q): \vec{p} \leqslant p \leqslant P_{u}\right\}$ 
where $M_{r}(p, q)=(p+h) \cdot \bar{X}(p)-(c+h) \cdot q+\cdots \cdot c$. We note that $(p+h) \cdot \bar{X}(p)$ is increasing on $\left[P_{l}, \bar{P}_{h}\right]$ and decreasing on $\left[\bar{P}_{h}, P_{t}\right]$. Moreover, $q \leqslant \bar{X}\left(\bar{P}_{h}\right) \Leftrightarrow \bar{p} \geqslant \bar{P}_{h}$.

It follows from the above discussion that

$$
M_{r}^{*}(q)= \begin{cases}\left(P_{u}+s-c\right) \cdot q-s \cdot \bar{X}\left(P_{u}\right)+c \cdot i & q \leqslant \bar{X}\left(P_{u}\right) \\ (\bar{p}-c) \cdot q+c \cdot i & \bar{X}\left(P_{c}\right) \leqslant q \leqslant \bar{X}\left(\bar{P}_{h}\right) \\ -(c+h) \cdot q+\left(\bar{P}_{h}+h\right) \cdot \bar{X}\left(\bar{P}_{h}\right)+c \cdot i & \bar{X}\left(\tilde{P}_{h}\right) \leqslant q\end{cases}
$$

It is proven in the Appendix that $(\hat{j}-c) \cdot q$ is a pseudoconcave function of $q$ on $\left(\bar{X}\left(P_{u}\right), \bar{X}\left(P_{1}\right)\right.$, . Thus, the rcsult follows from (29).

From (29) it is clear that $\tilde{p}_{\mathrm{r}}=\bar{P}_{c}=\min \left\{\operatorname{mav}\left\{\tilde{P}_{c}, P_{l}\right\}, P_{u}\right\}$, where $P_{c}$ is the maxımızer of the riskless profit function $(p-c) \cdot \bar{X}(p)$, and $\tilde{q}_{\mathrm{r}}=\bar{X}\left(\bar{P}_{1}\right)$.

We have $\theta(p, q) \geqslant \theta_{\mathrm{r}}(p, q)$ from (7). Thus, it follows from $(9)$ that $\bar{M}(p, q) \leqslant M_{\mathrm{r}}(p, q)$ which implics

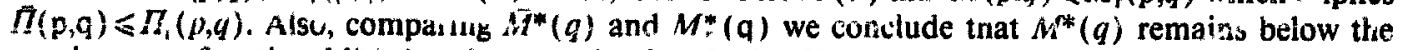
quasiconcave function $M_{r}^{*}(q)$ and approaches it at both tails.

\subsection{Additive modt}

Let $G(\cdot)$ be the distribution of $₫$, then $F(x ; p)=G(x-\bar{X}(p))$ which implics that

$\frac{\partial F(x ; p)}{\partial p}=-f(x ; p) \frac{\mathrm{d} X \bar{X}(p)}{\mathrm{d} p}$ and $\frac{\partial \Theta(p, q)}{\partial p}=-F(u ; p) \frac{\mathrm{d} \bar{X}(p)}{\mathrm{d} p}$

W:th these results, the expressions (12) through (15) cuuld be modified

Since $\tilde{p}$ is the opt mal price at $\tilde{q}$, it must satisfy the lirst order condition

$\left.\frac{\partial M(p, \tilde{q})}{\partial p}\right|_{j}=\tilde{q}-\theta(\tilde{p}, \tilde{q})+\left.(\tilde{p}-\hat{c}) \cdot \frac{\mathrm{d} \bar{X}(p)}{\mathrm{d} p}\right|_{p}=0$

which implies that $\bar{p}>c$. By adding anci subtracting $\bar{X}(\tilde{p}),(3 U)$ becomes

$\tilde{q}-\theta(\tilde{p}, \tilde{q})-\bar{X}(\tilde{p})+\left.\left(\bar{X}(0)+(p-c) \cdot \frac{\mathrm{d} \bar{X}(p)}{\mathrm{d} p}\right)\right|_{\hat{p}}=0$

By defintion, $\theta(p, q) \geqslant q-\bar{X}(p)$. Therefore, the expression in the brackets, which is the dernative of the riskless profit function, evaluated at $\tilde{p}$ must be positive. Thus, we conclude that $c \leqslant \tilde{p} \leqslant P_{c}$. This result was first proven by Mills [2] for a simple model. Karlin and Carr [3] showed that the same conclusion is true for the model we are studying by a different approach.

For a linear expected demand model and a uniform $\varepsilon$ on $[-\lambda, \lambda]$ we have $F(q ; p)=(q-a+b \cdot p+\lambda) /$ $2 \hat{i}$ and $\Theta(n, q)=\lambda \cdot \Gamma(q ; p)^{2}$ for all $q \in[\bar{X}(p)-\lambda, \hat{X}(p)+\lambda]$. Under this model, $\tilde{p}$ and $\tilde{q}$ must satisfy the first order conditions simultaneously (here, we ignore the presence of price bounds since the case of boundary solutions is trivial). These are given by

$\frac{\tilde{p}+s-c}{\tilde{p}+s+h}=\frac{\tilde{q}-a+b \cdot \tilde{p}+\lambda}{2 \cdot \lambda}$

añd

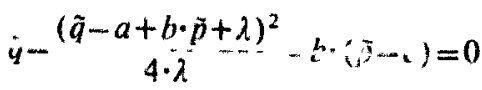

Solving (32) and $(2 ?)$ for $q$ we get

$\tilde{q}=\lambda \cdot\left(\frac{\tilde{p}+s-c}{\tilde{p}+s+\bar{h}}\right)^{2}+b \cdot(\tilde{p}-c)$ 
Moreover, by substituting (34) in (32) and arranging the terms we obtain

$2 \cdot(\tilde{p}+s+h)^{2} \cdot\left(P_{c}-\tilde{n}\right)-\lambda \cdot \frac{(h+c)^{2}}{h}=0$

which is a polynomial hoving a local maximum at $\left[2 \cdot p_{c}-(h+s)\right] / 3$. It fuliows that inis function has at least one and at most two positive roots. In addition, one of the roots is always located in the interval $\left(\left[2 \cdot P_{c}-(h+s)\right] / 3, P_{c}\right)$

Since the third citical point bo make a local minimum dury ncisxist, we conciuds that $M^{m}(q)$ is unimodal.

\subsection{Mailuphcailive model}

By definition, $F(x ; p)=G(x / \bar{X}(p))$, which implies that

$$
\frac{\partial F(x ; p)}{\partial p}=-x \cdot f(x ; p) \cdot(1 / \bar{X}(p)) \frac{\mathrm{d} \bar{X}(p)}{\mathrm{d} p}
$$

and

$$
\frac{\partial \Theta(p, q)}{\partial p}=-[q \cdot F(q ; p)-\Theta(p, q)] \cdot(1 / \bar{X}(p)) \frac{\mathcal{A}(p)}{\mathrm{d} p}
$$

With these resuits, the expressions (12) through (15) could be modified.

Evaluating (12) at $p_{4}$ and arranging toims we get

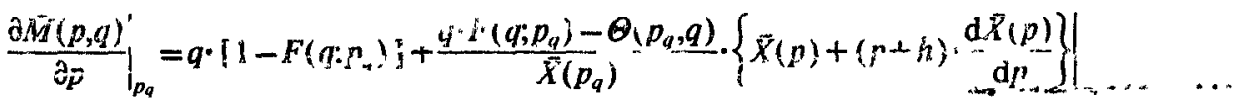

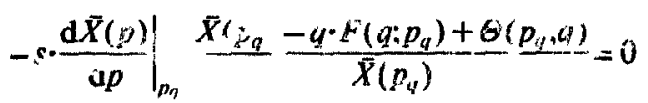

Since $\Theta(p, q) \geqslant q-\bar{X}(p)$, we have $\Theta(p, q)+\bar{X}(p)-q \cdot F(q ; p) \geqslant 0$. Thus, the first anc the third terms in (36) are positive. Moreover, we note that $q \cdot F(q p)-\theta(p, q) \geqslant 0$. Therefore, (36) impliec that

$\left.\left\{\bar{X}(p)+(p+h) \cdot \frac{\mathrm{d} \bar{X}(p)}{\mathrm{d} p}\right\}\right|_{r q q} \leqslant 0 \Leftrightarrow p_{q} \geqslant \bar{P}_{h}$

Furthermore, evaluating $(12) z^{t} ;$ and $\tilde{q}$ and ${ }^{\circ}$ earranging the terms we obtair

$$
\begin{aligned}
& \left.\frac{\tilde{q}}{\bar{X}(\tilde{p})} \cdot\left\{\bar{X}(p)+(p-c) \cdot \frac{\mathrm{d} \bar{X}(p)}{\mathrm{d} p}\right\}\right|_{p}-\left.\frac{\theta(\tilde{p}, \tilde{q})}{\bar{X}(\tilde{p})} \cdot\left\{\bar{X}(p)+(p+h) \cdot \frac{\mathrm{d} \bar{X}(p)}{\mathrm{d} p}\right\}\right|_{\tilde{r}} \\
& \cdot-\left.s \cdot \frac{\mathrm{d} \bar{X}(p)}{\mathrm{d} p}\right|_{\hat{p}} \cdot \frac{\bar{X}(\tilde{p})-\tilde{q}+\theta(\tilde{p}, \tilde{q})}{\bar{X}(\tilde{p})}=0
\end{aligned}
$$

The second term is positive, since $\tilde{p} \geq \vec{P}, n, j$ so is the third term. Thereforc, wa must have $\left.\left\{\bar{X}(p)+(p-c) \cdot \frac{\mathrm{d} \bar{X}(p)}{\mathrm{d} p}\right\}\right|_{p} \leqslant 0 \Leftrightarrow \tilde{p} \geqslant P,>c$

This result is the same as Karlin and Cary's [3] conclusion, which was proved by \& different approach than ours. 
If $\bar{X}(p)$ is linear and $\varepsilon$ has an exponcntial distribution, then the unimodality condition (28) reduces to

$$
2 \cdot \tilde{p}^{2}+(3 \cdot h+4 \cdot s-c) \cdot \tilde{p}+2(s+h) \cdot(s-c)-(h+c) \cdot \frac{a}{b} \geqslant 0
$$

The minimizer of the quadratic function in $(38)$ is $-(3 \cdot h+4 \cdot s-c) / 4$ which is less than $c$; hence, it is also l ss than $P_{c}$. It can be shown that if $a / b>c$, then the value of the quadratic function evaluated at $P_{c}$ is positive. Since $\tilde{p}>P_{c}$, this result implies condition (38). Therefore, $\tilde{M}^{*}(g)$ is unimodal for the exponential multiplicative demaud model. Zabel [4] arrived at the same conclusion, unaer some restrictions for the case where $s=0$.

\section{Conclusions}

There are analytical difficulties in verifying the unimodality of $\bar{M}^{*}(q)$ in a given problem. These arise mainly because $p_{q}$ or $\tilde{q}$ can not be explicitly evaluated. One possibility is to make simplifying assumptions so that analytical difficulties can be overcome. However, there is no majoi practical difficulty in testing these conditions numerically. We refer the reader to [8] for numerical esamples.

Since pricing decision affects the period ending inventory level, optixnality of $(\sigma, \Sigma)$ type policies for the multi-period model does not follow from the analysis of the one-period model. These issues are under current investigation.

\section{Appendix}

For $R(p)=p \cdot \bar{X}(p)$ we have

$$
\begin{aligned}
& R^{\prime}(p)=\bar{X}(p)+p \cdot \bar{X}^{\prime}(p) \\
& R^{\prime \prime}(p)=2 \cdot \bar{X}^{\prime}(p)+p \cdot \bar{X}^{\prime \prime}(p)
\end{aligned}
$$

Lemma A1. $R(p)$ is not pseudoconcave for all monotone decreasing $\bar{X}(p)$ functions.

Proof. If we let $\bar{X}(p)=600 \cdot \mathrm{e}^{-015 \nu}+1.5 \cdot \operatorname{Sin}(2 \cdot \pi \cdot p)$, which is a monotone decreasing function of $p$ on $(0,8)$, then $R(p)$ is not a pseudoconcave function on $(0,8)$.

Lemma A2. If $\bar{X}(p)$ is a convex decreasing function, then $R(p)$ is pseudoconcave on $(0, \infty)$.

Proof. Since $\bar{X}(p)$ is a convex ciecreasing function, $\forall p, p_{1} \in(0, \infty)$ we have

$\tilde{X}\left(p_{1}\right)-\tilde{X}(p) \geqslant\left(p_{1}-p\right) \cdot X^{\prime}\left(p_{1}\right)$

By definition, $R(p)$ will be pseudoconcave at $p_{1} \in(0, \infty)$ if it is differentidble at $p_{1}$ and

$R^{\prime}\left(p_{1}\right) \cdot\left(p-p_{1}\right) \leqslant 0 \Rightarrow R(p) \leqslant R\left(p_{1}\right), \forall p \in(0, \infty)$

Using (39) and (41) in (42) and arranging terms we get

$R^{\prime}\left(p_{1}\right) \cdot\left(p-p_{1}\right) \leqslant 0 \Rightarrow R(p)+\left(p-p_{1}\right) \cdot\left[\bar{X}\left(p_{1}\right)-\bar{X}(p)\right] \leqslant R\left(p_{1}\right) \rightarrow R(p) \leqslant R\left(p_{1}\right)$

Since $p_{1}$ was arbitrary the proof is valid for all $p_{1} \in(0, \infty)$.

Theorem A1. If $\bar{X}(p)$ is a convex or concave decreasing function, then $R(p)$ is pseudocowsave on $(0, \infty)$. 
Proof. If $\bar{X}(p)$ is concave, then from $(4 c$, it follows that $R(p)$ is concave on $(0, \infty)$. Also by Lemma $A 2, R(p)$ is pse doconcave on $(0, \infty)$ for a convex decreasing function.

Corollary A1. The function $T(p)=(p+a) \cdot \bar{X}(p)$ is pseudoconcave on $(0, \infty)$, where $a \in \mathbb{R}$.

Proof. Making a coordinate change by $p_{2} \leftarrow p+a$ and introducing the function $Y\left(p_{2}\right)=\bar{X}\left(p_{2}-a\right)$ we obtain $T(p)=(\bar{p}-a) \cdot \bar{X}(p)=p_{2} \cdot Y\left(p_{2}\right)$. By Theorem Al, $p_{2} \cdot Y\left(p_{2}\right)$ is pseudoconcave on $(a, \infty)$ which implies that $T(p)$, being a translation of $p_{2} \cdot Y\left(p_{2}\right)$, is pseudoconcave on $(0, \infty)$.

Corollary A2. $(p-c) \cdot q$ is a pseudoconcave function of $q$ on $\left(\bar{X}\left(P_{u}\right), \bar{X}\left(P_{f}\right)\right)$, where $q=\bar{X}(p)$.

Proof. $\bar{X}(p)$ is a decreasing function of $p$. Therefore, its inverse, $\bar{X}^{-1}(q)$, is decreasing on $\left(\bar{X}\left(P_{u}\right)\right.$, $\left.\bar{X}\left(P_{l}\right)\right)$. By Theorem Al, $q \cdot \bar{X}^{-1}(q)$ is pseudoconcave on $\left(\bar{X}\left(P_{u}\right), \quad \bar{X}\left(P_{l}\right)\right)$. Thus, $q \cdot \bar{X}^{-1}(q)-c \cdot q=(p-c) \cdot q$ is also pseudoconcave on $\left(\bar{X}\left(P_{u}\right), \bar{X}\left(P_{l}\right)\right)$.

\section{References}

1 Whitın, T M., 1955. Inventory control and price theory, Management Scl., 2. 61-68.

2 Mills, E.S., 1959. Uncertainty and price thcory. Quart. J. Econom., 73: 116-130.

3 Karlın, S and Carr, R.C., 1962. Prices and optımal ınventory polıcy. In: K.J. Arrow, S. karlın and H. Scarf (Eds.), Studies in Applied Probability and Management Science, Chapter 10. Stanford University Press, Stanford, CA.

4 Zabel, E., 1970. Monopoly and uncertainty. Rev. Economıc Stud, 37: 205-219.

5 Zabel, E., 1972. Multıperiod monopoly under uncertanty J. Econom Stud, 5 524-536.

6 Young, L., 1978. Price, inventory and the structure of uncertain demand New Zealand J. Oper. Res., 6 157-177.

7 Mills, E \$, 1962 Price, Output, and Inventory Policy Wiley, New York

8 Lau, A Hing-ing and Lau, Hon-Shiang, 1988 The newsboy problem with price-dependent demand distribution. IIE Trans, 20. $168-175$ 